\title{
The uncertainty of the experimentally-measured momentum coefficient
}

\author{
Guidelines on how to accurately estimate $C_{\mu}$ for a jet blowing through a thin slot
}

Richard Semaan $^{1}$ (1)

Received: 6 May 2020 / Revised: 8 October 2020 / Accepted: 15 October 2020 / Published online: 8 November 2020

(c) The Author(s) 2020

\section{Abstract}

We quantify the uncertainty of the momentum coefficient, $C_{\mu}$, for six different experimental approaches. The approaches vary depending on compressibility effects and on the utilized acquisition equipment to measure the product of the mass flow rate with the jet exit velocity. The uncertainty of the directly-measured variables is propagated into the momentum coefficient using the Taylor expansion method to the first order. All relevant random and systematic uncertainties are meticulously quantified and listed. The analysis reveals unacceptably high uncertainty of the momentum coefficient under certain settings. Practical solutions to minimize the sources of uncertainty are then proposed and analyzed. The proposed improvements on the benchmark example of a Coanda actuator with a high aspect ratio slot reduce the uncertainty of $C_{\mu}$ significantly but not sufficiently, as it remains at a non-negligible value of $\approx 11 \%$ for the best scenario. Finally, a list of practical recommendations and guidelines on how to accurately estimate the momentum coefficient experimentally is provided.

Richard Semaan

r.semaan@tu-bs.de

1 Institute of Fluid Mechanics, Technische Universität

Braunschweig, Hermann-Blenk-Str. 37, 38108 Brunswick,

Germany 


\section{Graphic abstract}

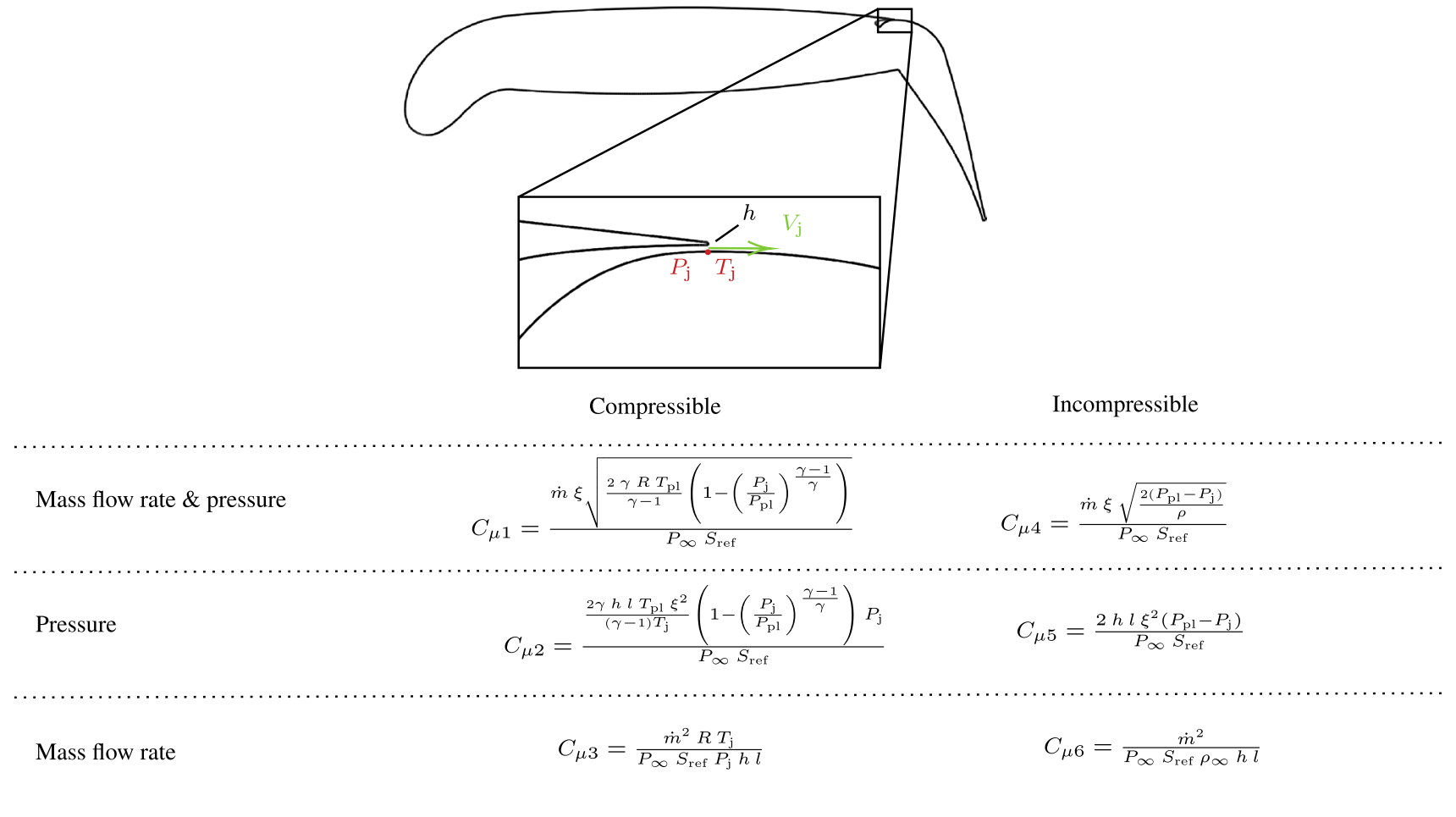

\section{Introduction}

The momentum coefficient, $C_{\mu}$, is the most common metric to quantify the blowing intensity of fluidic actuators (Cattafesta and Sheplak 2011; Kumar and Alvi 2006; Radespiel et al. 2016), such as fluidic oscillators (Viets 1975; Raman et al. 1993), tangential blowing (Pastoor et al. 2008) and Coanda actuators (El Sayed et al. 2017). Since its introduction by Poisson-Quinton and Lepage (1961), the momentum coefficient definition has remained practically unchanged. One exception is the work of Kelly (1956), who generalized the definition to cover both under- and over-expanded jet blowing as,

$C_{\mu}=\frac{\dot{m} V_{\mathrm{j}}+S_{\mathrm{j}}\left(P_{\mathrm{j}}-P_{\mathrm{a}}\right)}{\frac{1}{2} \rho_{\infty} V_{\infty}^{2} S_{\mathrm{ref}}}$,

where $\dot{m}$ is the mass flow rate, $V_{\mathrm{j}}, P_{\mathrm{j}}$, and $S_{\mathrm{j}}$ are the jet exit velocity, pressure, and area, respectively, $P_{\mathrm{a}}$ is the pressure near the jet exit, the subscript $\infty$ denotes freestream conditions, and $S_{\text {ref }}$ is a reference area typically defined for airfoils as $S_{\text {ref }}=c \eta$, where $c$ is the chord length and $\eta$ is the span. Physically, the momentum coefficient can be interpreted as the normalized jet thrust (or drag).
Due to its importance in quantifying the blowing intensity, and correspondingly the actuation effectiveness, and due to its small value (typically $\left.\mathcal{O}\left(10^{-3}\right)-\mathcal{O}\left(10^{-2}\right)\right)$ compared to other aerodynamic coefficients such as the lift (typically $\mathcal{O}\left(10^{-1}\right)$ ), it is critical to accurately estimate $C_{\mu}$. This issue is exasperated when reporting aerodynamic benefits, such as the lift gain ratio $\Delta C_{l} / C_{\mu}$, where small errors in estimating $C_{\mu}$ will yield large uncertainties in the actuation gains.

Several approaches to experimentally estimate the momentum coefficient have been reported in the literature (Englar 2000; El Sayed et al. 2017). Examples range from solely relying on mass flow rate measurements (Scholz et al. 2008) to using only pressure measurements (Oswald et al. 2019; Shaqarin et al. 2019). Experimental challenges in estimating $C_{\mu}$ have already been reported by El Sayed et al. (2018). However, no rigorous uncertainty analysis on the various possible momentum coefficient estimation approaches has been conducted. Moreover, there exist no clear guidelines on how to best experimentally measure $C_{\mu}$. In this study, we perform a comprehensive analysis of 


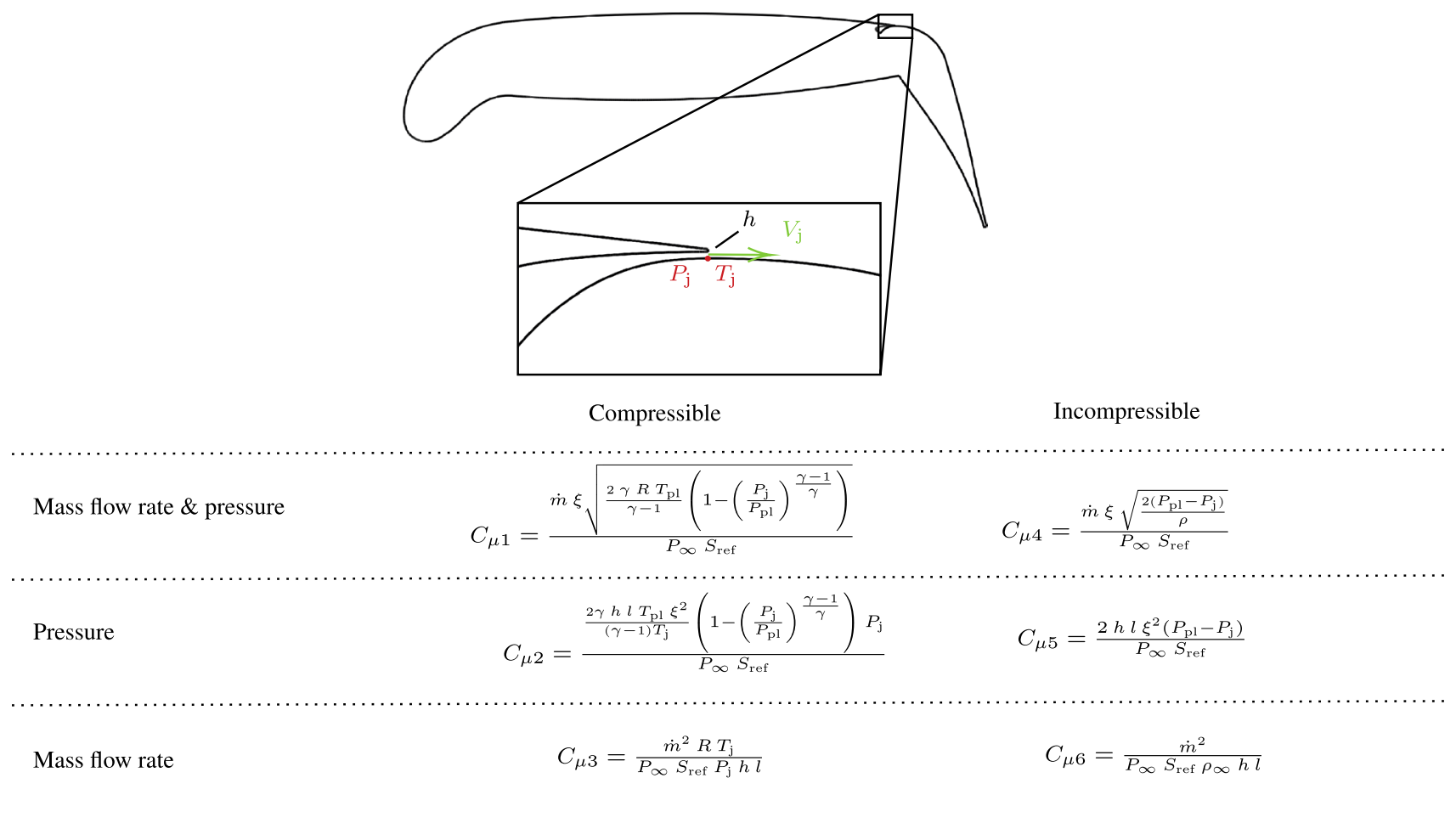

Fig. 1 Summary of the six momentum coefficient experimental estimation approaches for both compressible and incompressible flows. Also shown is the benchmark high-lift setup used and a zoom-in on the jet exit region with some of the variables

the various approaches to estimate the momentum coefficient, and we quantify their corresponding uncertainty. As a benchmark example for the current analysis, we employ Coanda actuation (Englar 2000; El Sayed et al. 2017) as a fluidic actuator, which includes a range of experimental uncertainty sources. We rely on numerical simulation of the flow (Semaan et al. 2016; François et al. 2018) and on two experimental measurements: one in the wind tunnel (El Sayed et al. 2018) and one in the water tunnel (El Sayed et al. 2017). The uncertainty analysis is performed using the Taylor-based moment propagation method (Ku 1966) to the first order. Based on this analysis, we provide recommendations and best practices on how to most accurately measure the momentum coefficient.

The manuscript is structured as follows. The various experimental methods to estimate the momentum coefficient are presented in Sect. 2 . The uncertainty propagation method, as well as the quantification of the various random and systematic uncertainties, are detailed in Sect. 3. In Sect. 4, the results are presented and the uncertainty of the various approaches is discussed. A summary of the work and recommendations on how to best determine $C_{\mu}$ are provided in Sect. 5. The details of the numerical simulation are presented in Appendix 1. The two experimental setups are detailed in Appendix 2.

\section{Experimental approaches to estimate the momentum coefficient}

Depending on the measurement equipment, on compressibility effects, and the jet exit velocity, various approaches exist to estimate the momentum coefficient. Each approach yields a different version of equation (1). Owing to the current lack of experimental data in the sonic and supersonic regime, we exclusively focus in this study on blowing with subsonic jet exit velocity. Our actuator design, and subsequently all our experimental measurements during the project are performed in the subsonic range, which implies $P_{\mathrm{j}}=P_{\mathrm{a}}$, and simplifies equation (1) to the original momentum coefficient definition of Poisson-Quinton and Lepage (1961),

$C_{\mu}=\frac{\dot{m} V_{\mathrm{j}}}{\frac{1}{2} \rho_{\infty} V_{\infty}^{2} S_{\text {ref }}}$. 
It is worth to note that the neglected sonic and supersonic jet exit velocity regimes require the additional consideration of the uncertainty of $P_{\mathrm{a}}$, which is often non-negligible due to the difficulty in measuring it experimentally. The second relevant uncertainty to consider for those flow regimes is that of the jet exit area $S_{\mathrm{j}}$, which we investigate in Sect. 3.3.2.

Uncertainty analysis requires expressing the analytical expression as a function of the directly measured variables. As such, the dynamic pressure expressed as $\frac{1}{2} \rho_{\infty} V_{\infty}^{2}$ is substituted by its directly-measured value $P_{\infty}$ (sometimes referred to as $q_{\infty}$ ) from a pitot Prandtl tube installed at the wind or water tunnel test section entrance, yielding,

$C_{\mu}=\frac{\dot{m} V_{\mathrm{j}}}{P_{\infty} S_{\mathrm{ref}}}$.

For the freestream dynamic pressure $P_{\infty}$, we neglect all uncertainties associated with the angular positioning of the probe, which are usually very small after careful setup.

The main differences in experimentally estimating $C_{\mu}$ lie in the approaches to measure the numerator $\dot{m} V_{\mathrm{j}}$ in equation (3). In this section, we present six different approaches to estimate $C_{\mu}$ and their corresponding definitions. For each approach, we report the necessary measurement equipment. All six approaches are schematically summarized in Fig. 1. The approaches distinguish between compressible and incompressible jets, and among three different experimental methods to measure $\dot{m} V_{\mathrm{j}}$. We intentionally exclude the scenario of direct $V_{\mathrm{j}}$ measurement during the measurement campaign (e.g. using a hot-wire), since it is deemed unpractical and inaccurate. A hot-wire setup at the jet exit will disturb the flow and diminishes the Coanda effect. In this study, we only consider steady blowing, which excludes dynamic effects and significantly simplifies the uncertainty analysis.

\subsection{Compressible jet flow}

Owing to the small slot height and to the high contraction ratio of the nozzle, the jet exit velocity lies typically in the compressible range. As we will see in the following, this necessitates measurement of either the jet exit temperature $T_{\mathrm{j}}$, the plenum temperature $T_{\mathrm{pl}}$, or of both. In this study, we assume similar uncertainty between the two temperature measurements, i.e., $u^{T_{\mathrm{j}}} \approx u^{T_{\mathrm{pl}}}$. We emphasize, this assumption does not assert that $T_{\mathrm{j}} \approx T_{\mathrm{pl}}$, but rather that their uncertainty is similar. In other words, the two variables are treated independently, and their uncertainties are propagated individually. Only the magnitude of their respective relative uncertainty is assumed similar. This is a reasonable assumption, since the temperature change between the plenum and the jet exit is not typically very large, and the employed temperature measurement equipment is usually the same.
Another motivation for this assumption stems from the experimental difficulty to measure the temperature at the jet exit, which constitutes a limitation to any approach relying on the jet exit temperature, such as $C_{\mu 2}$ and $C_{\mu 3}$. In this study, we assume that the jet exit pressure $P_{\mathrm{j}}$ is measurable, whereas the jet exit temperature $T_{\mathrm{j}}$ is not. This is since $T_{\mathrm{j}}$ is harder to measure than $P_{\mathrm{j}}$, where a long tube that conducts the pressure signal to a sensor situated at some distance from the exit can be used.

\subsubsection{Combined measurements}

The most common approach to experimentally estimate the momentum coefficient relies on the direct measurement of the mass flow rate and the plenum and jet exit static pressures. We refer to this as the combined measurement approach. The pressure measurements are used to compute the jet exit velocity via isentropic expansion approximation assuming a constant jet velocity across the slot height and a sufficiently large plenum with a quasi-zero flow velocity $V_{\mathrm{pl}} \approx 0 \mathrm{as}$,

$V_{\mathrm{j}}=\xi \sqrt{\frac{2 \gamma R T_{\mathrm{pl}}}{\gamma-1}\left(1-\left(\frac{P_{\mathrm{j}}}{P_{\mathrm{pl}}}\right)^{\frac{\gamma-1}{\gamma}}\right)}$,

where $\xi$ is the nozzle efficiency factor. For a sufficiently large plenum volume and large slot aspect ratio, the constant jet velocity assumption across the exit slot is reasonable, as reported in the literature from experimental measurements, e.g., Englar et al. (2009). Moreover, deviations from the ideal constant velocity profile at the exit are mostly accounted for with the viscous losses term $\xi$. The nozzle efficiency factor $\xi$ can be estimated from numerical simulations, from existing catalogs, or, at the risk of increased uncertainty, by simply equating $C_{\mu 1}$ to $C_{\mu 3}$ (or $C_{\mu 4}$ to $C_{\mu 6}$ for incompressible flows). Alternatively, and particularly for complex nozzle geometries, $\xi$ can be accurately estimated before the start of the measurement campaign by carefully measuring the jet exit velocity distribution using, for example, a hot-wire. Due to the multitude of available options to accurately estimate $\xi$, it is reasonable to assume its uncertainty to be negligible. In the current study, $\xi$ is estimated from scale-resolving numerical simulations (Semaan et al. 2016), which yield $\xi \approx 0.96$. Substituting the jet exit velocity expression (4) in equation (3) yields,

$C_{\mu 1}=\frac{\dot{m} \xi \sqrt{\frac{2 \gamma R T_{\mathrm{pl}}}{\gamma-1}\left(1-\left(\frac{P_{\mathrm{j}}}{P_{\mathrm{pl}}}\right)^{\frac{\gamma-1}{\gamma}}\right)}}{P_{\infty} S_{\mathrm{ref}}}$. 


\subsubsection{Pressure measurements}

The second approach relies on the pressure measurements in the plenum and at the jet exit to infer the jet exit velocity from isentropic expansion [equation (4)]. The mass flow rate is expressed as function of $V_{\mathrm{j}}$ based on conservation of mass and the ideal gas law as,

$\dot{m}=\rho_{\mathrm{j}} l h V_{\mathrm{j}}=\frac{P_{\mathrm{j}}}{R T_{\mathrm{j}}} h l V_{\mathrm{j}}$,

where $h$ is the jet slot height, and $l$ is the slot span. This yields,

$C_{\mu 2}=\frac{\frac{2 \gamma h l T_{\mathrm{pl}} \xi^{2}}{(\gamma-1) T_{\mathrm{j}}}\left(1-\left(\frac{P_{\mathrm{j}}}{P_{\mathrm{pl}}}\right)^{\frac{\gamma-1}{\gamma}}\right) P_{\mathrm{j}}}{P_{\infty} S_{\mathrm{ref}}}$.

This approach is better suited for experiments with unsteady blowing than the combined (Sect. 2.1.1) and the mass flow rate approach (Sect. 2.1.3), since flowmeters have slow response time making real-time $C_{\mu}$ estimation impossible. We reiterate the experimental difficulties in measuring $T_{\mathrm{j}}$, particularly for complex geometries. One possible approach to acquire $T_{\mathrm{j}}$ is to measure it for a range of expected pressure ratios before the start of the measurement campaign.

\subsubsection{Mass flow rate measurements}

The final approach for compressible jet flows employs only mass flow rate measurements to estimate $\dot{m} V_{\mathrm{j}}$. The jet exit velocity is calculated from the mass flow rate using conservation of mass. Equation (3) becomes,

$C_{\mu 3}=\frac{\dot{m}^{2} R T_{\mathrm{j}}}{P_{\infty} S_{\mathrm{ref}} P_{\mathrm{j}} h l}$.

Despite only relying on mass flow measurements to estimate $\dot{m} V_{\mathrm{j}}$, equation (7) still depends on two additional measured quantities, $P_{\mathrm{j}}$ and $T_{\mathrm{j}}$, that also carry uncertainties. Both of these variables, particularly $T_{\mathrm{j}}$, could be challenging to measure.

\subsection{Incompressible jet flow}

The second category of momentum coefficient estimation approaches assumes incompressible jet flow. This category applies for experiments with low jet exit velocity, which could be due to a relatively large slot height, and for experiments performed in an incompressible medium such as water (El Sayed et al. 2017). Making this assumption should not be taken lightly.

Assuming incompressibility for a compressible flow might lead to significant errors, especially at elevated pressure ratios, as reported by El Sayed et al. (2018). For an incompressible flow, the jet density can be assumed identical to the freestream density $\rho_{\mathrm{j}}=\rho_{\infty}$. This relaxes the requirement to measure the temperature in the plenum and at the jet exit for all following three definitions.

\subsubsection{Combined measurements}

Similar to the compressible flow cases, estimating the momentum coefficient can be accomplished using three different approaches. The first combined approach employs mass flow rate and pressure measurements. As previously mentioned, no temperature measurements are required for incompressible flows. Under these assumptions, one can use the Bernoulli equation between the plenum and the jet exit to compute the jet exit velocity as,

$V_{\mathrm{j}}=\xi \sqrt{\frac{2\left(P_{\mathrm{pl}}-P_{\mathrm{j}}\right)}{\rho}}$,

which upon substitution in equation (3) yields,

$C_{\mu 4}=\frac{\dot{m} \xi \sqrt{\frac{2\left(P_{\mathrm{pl}}-P_{\mathrm{j}}\right)}{\rho}}}{P_{\infty} S_{\mathrm{ref}}}$.

\subsubsection{Pressure measurements}

The second approach for incompressible flows assumes the availability of only pressure measurements for $\dot{m} V_{\mathrm{j}}$ estimation. The mass flow rate is expressed as function of $V_{\mathrm{j}}$ based on the conservation of mass yielding,

$C_{\mu 5}=\frac{2 h l \xi^{2}\left(P_{\mathrm{pl}}-P_{\mathrm{j}}\right)}{P_{\infty} S_{\mathrm{ref}}}$.

\subsubsection{Mass flow rate measurements}

The final approach measures the mass flow rate and estimates $V_{\mathrm{j}}$ from the conservation of mass to yield,

$C_{\mu 6}=\frac{\dot{m}^{2}}{P_{\infty} S_{\text {ref }} \rho_{\infty} h l}$.

For this incompressible flow, the flow density $\rho_{\infty}$ is typically accurately estimated from temperature measurement in the test section. We, therefore, assume it has negligible uncertainty, $u^{\rho_{\infty}} \approx 0$. 
Table 1 The uncertainty magnification factors (UMF) for the six momentum coefficient definitions

\begin{tabular}{|c|c|c|c|c|c|c|}
\hline & $C_{\mu 1}$ & $C_{\mu 2}$ & $C_{\mu 3}$ & $C_{\mu 4}$ & $C_{\mu 5}$ & $C_{\mu 6}$ \\
\hline$\dot{m}$ & 1 & N/A & 2 & 1 & N/A & 2 \\
\hline$T_{\mathrm{pl}}$ & $\frac{1}{2}$ & 1 & N/A & N/A & N/A & N/A \\
\hline$T_{\mathrm{j}}$ & N/A & -1 & 1 & N/A & N/A & N/A \\
\hline$P_{\mathrm{j}}$ & $\frac{(\gamma-1) P_{\mathrm{j}}}{2 \gamma\left(P_{\mathrm{j}}-P_{\mathrm{pl}}\left(\frac{P_{\mathrm{j}}}{P_{\mathrm{pl}}}\right)^{\frac{1}{\gamma}}\right)}$ & $\frac{(2 \gamma-1) P_{\mathrm{j}}-\gamma\left(\frac{P_{\mathrm{j}}}{P_{\mathrm{pl}}}\right)^{\frac{1}{\gamma}} P_{\mathrm{pl}}}{\gamma\left(P_{\mathrm{j}}-P_{\mathrm{pl}}\left(\frac{P_{\mathrm{j}}}{P_{\mathrm{pl}}}\right)^{\frac{1}{\gamma}}\right)}$ & -1 & $-\frac{P_{\mathrm{j}}}{2\left(P_{\mathrm{pl}}-P_{\mathrm{j}}\right)}$ & $-\frac{P_{\mathrm{j}}}{\left(P_{\mathrm{pl}}-P_{\mathrm{j}}\right)}$ & N/A \\
\hline$P_{\mathrm{pl}}$ & $-\frac{(\gamma-1) P_{\mathrm{j}}}{2 \gamma\left(P_{\mathrm{j}}-P_{\mathrm{pl}}\left(\frac{P_{\mathrm{j}}}{P_{\mathrm{pl}}}\right)^{\frac{1}{\gamma}}\right)}$ & $-\frac{(\gamma-1) P_{\mathrm{j}}}{\gamma\left(P_{\mathrm{j}}-P_{\mathrm{pl}}\left(\frac{P_{\mathrm{j}}}{P_{\mathrm{pl}}} \frac{1}{\gamma}\right)\right.}$ & N/A & $\frac{P_{\mathrm{pl}}}{2\left(P_{\mathrm{pl}}-P_{\mathrm{j}}\right)}$ & $\frac{P_{\mathrm{pl}}}{\left(P_{\mathrm{pl}}-P_{\mathrm{j}}\right)}$ & N/A \\
\hline$P_{\infty}$ & -1 & -1 & -1 & -1 & -1 & -1 \\
\hline$h$ & N/A & 1 & -1 & N/A & 1 & -1 \\
\hline
\end{tabular}

N/A not applicable

\section{Uncertainties quantification}

The uncertainty quantification is central to the current work. In this section, we present the uncertainty propagation method in Sect. 3.1, which yields the uncertainty magnification factors that are discussed in Sect. 3.2. Our endeavor to quantify the individual random and systematic uncertainty of the measured variables is presented in Sect. 3.3. In Sect. 3.4, we summarize all assumptions made in Sect. 2 and 3.

\subsection{Taylor expansion method for uncertainty propagation}

The uncertainties of the dependent variables are propagated to $C_{\mu}$ using the Taylor expansion method (Ku 1966) to the first order. This approach is practical for problems that are not highly nonlinear, such as the current one, as demonstrated in the uncertainty magnification factor distributions in Sect. 3.2 and Fig. 2. It is simpler than Monte Carlo methods and has the additional benefit of delivering the contribution of each variable to the result uncertainty, i.e., to the momentum coefficient. In the following, we briefly describe the method. For more details, the reader is referred to existing literature on the subject (Ku 1966; Du and Chen 2002; Padulo et al. 2007).

As we have learned in Sect. 2, the momentum coefficient depends on different uncertain measured variables that change with the approach. A general formulation of the momentum coefficient can be expressed as,

$C_{\mu}=C_{\mu}\left(x_{1}, x_{2}, \ldots, x_{I}\right)$,

where $x_{i}$ are the uncertain directly measured variables, and $I$ is the total number of variables for each approach.

The uncertainties of $x_{i}$ are propagated to the momentum coefficient through equation (11), which is referred to as the data reduction equation (DRE) (Coleman and Steele 2009).
We quantify this propagated uncertainty using the Taylor expansion method to the first order as,

$$
\begin{aligned}
u_{C_{\mu}}^{2}= & \sum_{i=1}^{I}\left(\frac{\partial C_{\mu}}{\partial x_{i}}\right)^{2} u_{x_{i}}^{2} \\
& +2 \sum_{i=1}^{I-1} \sum_{j=i+1}^{I}\left(\frac{\partial C_{\mu}}{\partial x_{i}}\right)\left(\frac{\partial C_{\mu}}{\partial x_{j}}\right) u_{x_{i}, x_{j}},
\end{aligned}
$$

where $u_{x_{i}}$ is the uncertainty of the mean measured variable $x_{i}$, and $u_{x_{i}, x_{j}}$ denotes the correlated uncertainty between variable $x_{i}$ and $x_{j}$. For cases where all measured variables are statistically independent, the second summation term becomes zero. Here, for simplicity we write $C_{\mu}$ and $u_{C_{\mu}}$ instead of $\bar{C}_{\mu}$ and $u_{\bar{C}_{\mu}}$ to refer to the mean momentum coefficient and its corresponding uncertainty. In this study, we are only interested in the uncertainty of the mean momentum coefficient and not in its higher-order moments. All propagated uncertainties are, therefore, those of the mean values.

Equation (12) is the most general expression for the propagation of uncertainty. It assumes a correlation among all variables. As will be discussed in Sect. 3.3, only two measured variables are correlated in the current study, the lip height and the jet exit pressure.

It is more practical to express the uncertainty in relative terms. As such, equation (12) can be expressed as (neglecting the correlation term),

$\left(\frac{u_{C_{\mu}}}{C_{\mu}}\right)^{2}=\sum_{i=1}^{I}\left(\frac{x_{i}}{C_{\mu}} \frac{\partial C_{\mu}}{\partial x_{i}}\right)^{2}\left(\frac{u_{x_{i}}}{x_{i}}\right)^{2}$.

This formulation has the added benefit of yielding the so called uncertainty magnification factors (UMF),

$\mathrm{UMF}_{i}=\frac{x_{i}}{C_{\mu}} \frac{\partial C_{\mu}}{\partial x_{i}}$ 
which reflect how the uncertainty in each variable is magnified through equation (11).

Equations (12) and (13) can be used to propagate either random or systematic uncertainty, which are then combined to form the combined standard uncertainty of the mean as,

$u=\sqrt{\sigma_{X}^{2}+b^{2}}$,

where $\sigma_{X}$ is the standard deviation of the mean,

$\sigma_{X}=\frac{\sigma}{\sqrt{N}}$

where $\sigma$ is the standard deviation, and $N$ is the number of independent samples. $b$ is the total systematic uncertainty of a measured variable $x_{i}$. It is computed as,

$b=\sqrt{\sum_{j=1}^{J} b_{j}^{2}}$,

where the $b_{j}$ 's are $J$ different systematic uncertainties affecting $x_{i}$. In the current study we present all uncertainties with 95\% confidence level, which is the norm in the engineering sciences. It is typical to report the uncertainty using the combined expanded uncertainty $U=2 u$, where $u$ is the combined standard uncertainty of the mean defined in equation (15). The factor 2 is based on the large-sample record assumption for $95 \%$ confidence level. The expanded uncertainty can be also understood as the uncertainty margins of the mean value.

Following Coleman and Steele (2009), we additionally define the uncertainty percentage contribution (UPC) as

$\mathrm{UPC}_{i}=\frac{\left[\left(x_{i} / C_{\mu}\right)\left(\partial C_{\mu} / \partial x_{i}\right)\right]^{2}\left(U_{x_{i}} / x_{i}\right)^{2}}{\left(U_{C_{\mu}} / C_{\mu}\right)^{2}}$,

which yields the percentage contribution of the uncertainty in a variable $x_{i}$ to the squared uncertainty in the momentum coefficient. The UPCs contain the UMF effects as well as the individual uncertainties.

\subsection{Uncertainty magnification factors}

The UMF for a given measured variable $x_{i}$ indicates the influence of the uncertainty in that variable on the uncertainty in the momentum coefficient. An absolute UMF value greater than 1 indicates that the influence of the uncertainty in the variable is magnified as it propagates through the data reduction equation into $C_{\mu}$. An absolute UMF value of less than 1 indicates that the influence of the uncertainty in the variable is diminished as it propagates through the data

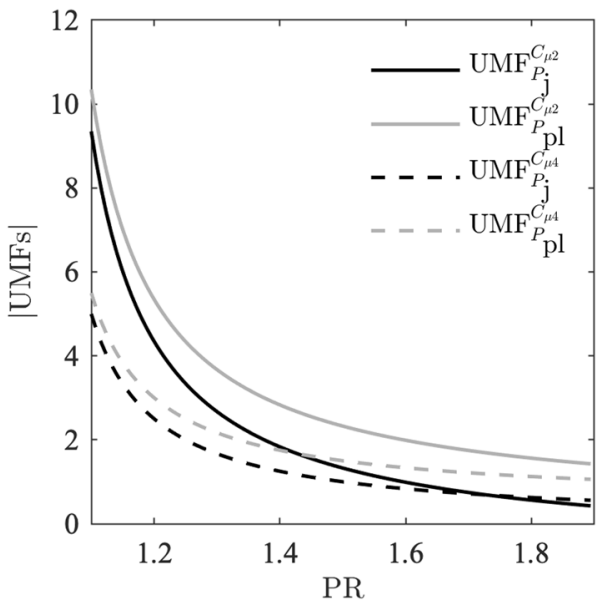

Fig. 2 The absolute value of the uncertainty magnification factors of $P_{\mathrm{j}}$ and $P_{\mathrm{pl}}$ for $C_{\mu 2}$ and $C_{\mu 4}$, respectively

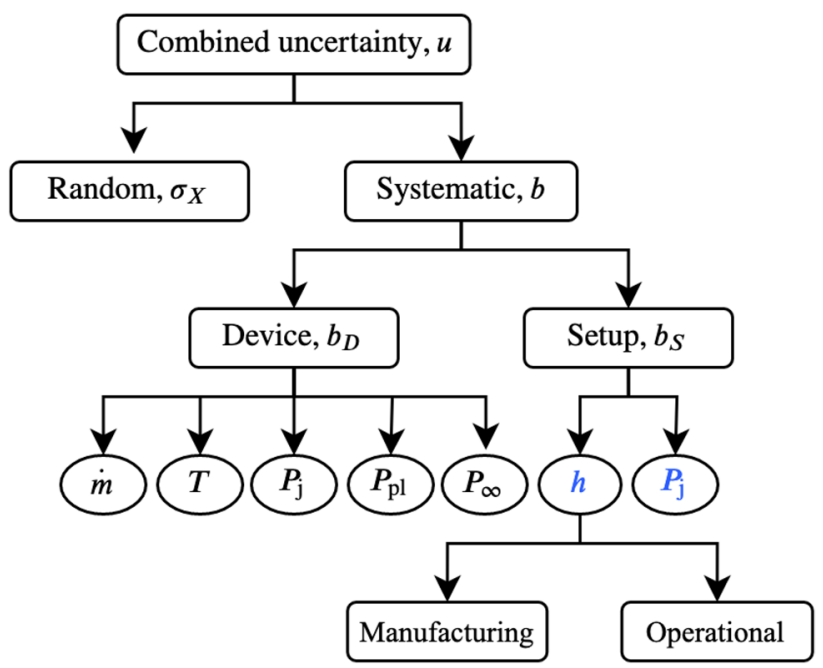

Fig. 3 A schematic summarizing all the random and systematic uncertainties relevant to the current problem. Blue denotes the correlated uncertainty between the lip height and jet exit pressure

reduction equation into $C_{\mu}$. Since the UMFs are squared in equation (13), their signs are of no relevance.

The UMF's derivation is manually performed for each of the six approaches in Sect. 2. The expressions are listed in Table 1.

As the table shows, not all UMFs are constants. From the constant UMFs, only one variable, $T_{\mathrm{pl}}$, has an uncertainty magnification factor less than one for $C_{\mu 1}$. All other variables have either a neutral (value $=1$ ) or a magnifying 
Table 2 Summary of the relative systematic, random, and total uncertainties

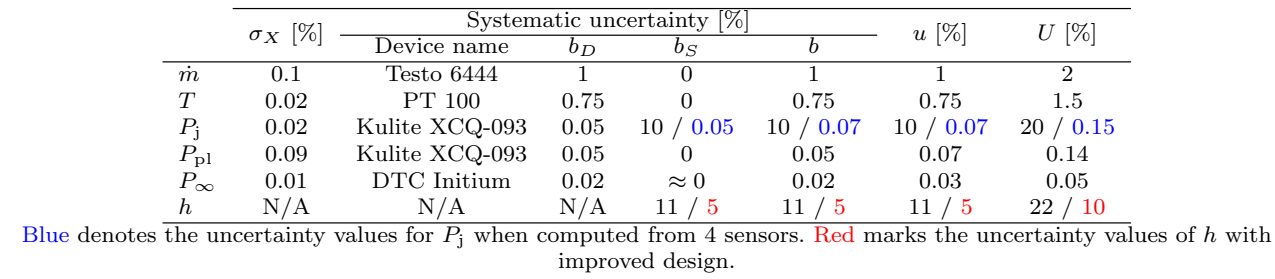

effect (value $>1$ ), which is that of $\dot{m}$ for $C_{\mu 3}$ and $C_{\mu 6}$, where only the mass flow rate is measured. This suggests that high uncertainty in the measurement of the mass flow rate will get magnified as it propagates into the data reduction equation (11).

The variable UMFs are those of $P_{\mathrm{j}}$ and $P_{\mathrm{p}}$, which in turn depend on $P_{\mathrm{j}}$ and $P_{\mathrm{pl}}$, or on the pressure ratio $\mathrm{PR}=\frac{P_{\mathrm{pl}}}{P_{\mathrm{j}}}$. Figure 2 shows the UMF variations of $P_{\mathrm{j}}$ and $P_{\mathrm{pl}}$ for $C_{\mu 2}$ and $C_{\mu 4}$. We refrain from showing those of $C_{\mu 1}$ and $C_{\mu 5}$ since they are linearly related to the presented ones (twice or half the values). The UMFs are presented for a realistic pressure ratio range between 1.1 and 1.894. $\mathrm{PR}=1.894$ delimits the value when the exit Mach number becomes critical. As the figure shows, the UMFs for $P_{\mathrm{j}}$ and $P_{\mathrm{pl}}$ are significantly magnified for low-pressure ratios. They asymptotically decrease with higher pressure ratios towards values below one, except for $\mathrm{UMF}_{P_{\mathrm{j}}}^{C_{\mu 2}}$ and $\mathrm{UMF}_{P_{\mathrm{pl}}}^{C_{\mu 2}}$ which remain larger than one in the investigated pressure ratio range. The results clearly show how pressure measurement uncertainties get significantly magnified at lower pressure ratios (i.e., blowing intensities). As detailed in the following section, efforts should be undertaken to reduce these uncertainties, particularly at low momentum coefficients.

\subsection{Random and systematic uncertainties}

In this section, we present the estimated relative random and systematic uncertainties of all the measured variables. The various types of uncertainties relevant to the current problem are schematically illustrated in Fig. 3. All uncertainty estimates are listed in Table 2. Random uncertainties of the mean variables $\sigma_{X}$ are straightforward to estimate. They are computed from experimental measurements as the standard deviation of the mean (equation (16)) using the in-house tool SCOUT (Semaan and Yadav 2020). As the table shows, all random uncertainties are low for the current investigation. This is mainly due to the long sample records acquired during the measurement campaigns.

Systematic uncertainties are more challenging to estimate. The sources of systematic uncertainty are manifold, such as calibration errors, data acquisition errors, or data reduction errors. In this study, we perform careful calibration on all equipment and we properly utilize all hardware following the manufacturer's recommendation, which

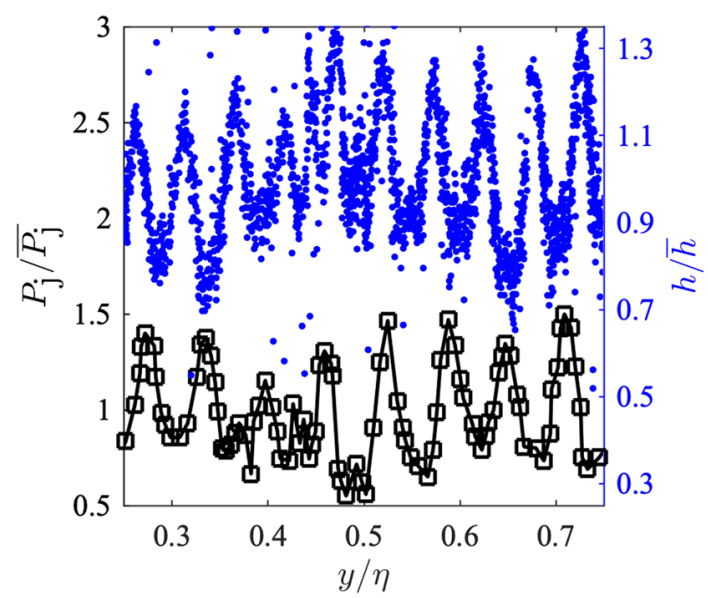

Fig. 4 Spanwise normalized jet exit pressure and slot height distributions for the wind tunnel model measured by a fish mouth pitot probe and a laser scanner, respectively (adapted from El Sayed et al. 2018)

reduces the calibration and data acquisition errors to negligible values.

We classify the remaining systematic uncertainties into two categories: device $\left(b_{D}\right)$ and setup $\left(b_{S}\right)$ uncertainties.

\subsubsection{Device systematic uncertainty}

The device uncertainty quantifies the accuracy of the measurement equipment. It is usually provided by the manufacturer as full-scale uncertainty. We note that the measurement equipment employed in the current investigations (listed in Table 2) are standard across the fluid mechanics' field. It is safe to assume that all standard alternative sensors have comparable systematic uncertainty. As Table 2 shows, the device systematic uncertainties are low, except for the mass flowmeter and the thermocouple. The flowmeter accuracy is provided by the manufacturer as $\pm 3 \%$ (the measured value) $\pm 0.3 \%$ (the full scale). For the current measurement range, this approximates to $b_{D}^{\dot{m}} \approx 1 \%$ (or $\pm 2 \%$ expanded uncertainty). To the author's knowledge, there exists no commercial flowmeter with significantly better accuracy. Unlike flowrate measurements, there is a large array of temperature measurement devices with varying accuracy. Typical PT100 thermocouples have a systematic accuracy that ranges between $0.75 \%$ and $0.075 \%$. For the current analysis, we assume the least accurate type with $b_{D}^{T}=0.75 \%$. 


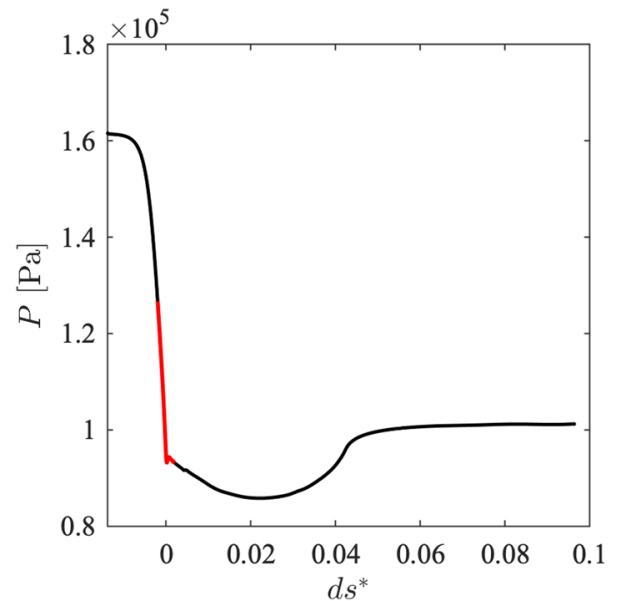

Fig. 5 The mean static pressure distribution over the model surface extending from inside the nozzle to the flap shoulder. $d s^{*}$ denotes normalized curvilinear deviations from the jet exit position. The red section marks the uncertain pressure range that stems from the $\pm 0.2 \mathrm{~mm}$ sensor positioning uncertainty. The results are numerically generated from Semaan et al. (2016)

\subsubsection{Setup systematic uncertainty}

As its name suggests, setup uncertainty stems from bias error during the preparation and the conduction of the experiment, such as misalignment or inaccurate sensor positioning. In the current study, two variables have relevant setup uncertainties: the slot height, and the jet exit pressure. As previously mentioned, the setup uncertainty of $P_{\infty}$, which stems from the angular positioning of the pitot Prandtl probe, is assumed negligible.

The first major systematic setup uncertainty is that of the slot height $h$. The slot of many fluidic actuators is typically a fraction of a millimeter high and extends across most of the model $\operatorname{span}^{1}$. This makes the slot length several orders of magnitude wider than its height. The uncertainty in $h$ originates from manufacturing/installation and from operational errors.

The manufacturing and installation errors can be estimated by accurately measuring the height distribution across the span. This can be achieved by laser-based scanning devices, which are usually much more accurate than feeler gauges. Figure 4 presents the slot height distribution of the Coanda blown flap in the wind tunnel experiment measured with gapCONTROL 2911-10/BL 3D Laser scanner before final adjustments. Also shown is the spanwise jet exit normalized pressure distribution, which will be detailed

\footnotetext{
1 This assumption does not apply for several fluidic actuator types, such as microjets (Kumar and Alvi 2006) and fluidic oscillators (Glezer and Amitay 2002). For these actuators, the setup uncertainty can be assumed negligible.
}

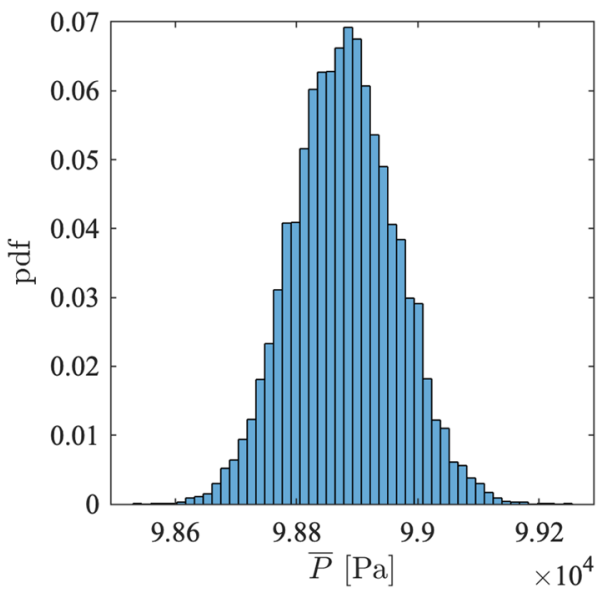

Fig. 6 The probability density function of the bootstrapped mean sampled from the uncertain sensor range

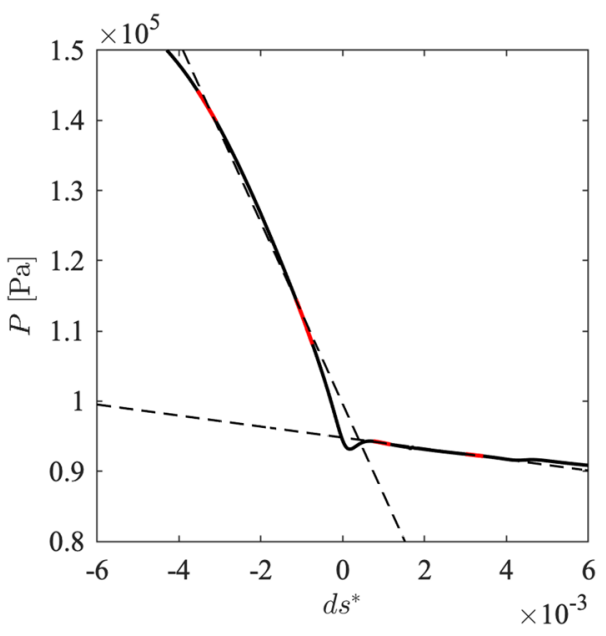

Fig. 7 The static pressure distribution around the jet exit. The red lines mark the uncertain range of the four pressure sensors used to infer the jet exit pressure at the intersection point of the two linear fits

below. The observed waviness in both distributions corresponds to the adjustment bolt locations. After careful adjustments, the lip height distribution uncertainty was reduced to $b_{S, 1}^{h} \approx 10 \%$ (not shown), which is the value we adopt in the first scenario.

It is worth to note that, besides increased uncertainty, lip height fluctuation and the resultant spanwise variation of the blowing intensity might, in severe cases, void the spanwise flow homogeneity assumption.

The systematic operational uncertainty originates from errors that occur during the experiment. The systematic operational uncertainty of $h$ stems from the deflection of the typically thin actuator lid under loading. The thinness is dictated from aerodynamic and conceptual constraints, where it is desired to preserve the model reference geometry while 
actuating tangentially to the model's surface. This thin construction makes the lid vulnerable to bending. The amount of bending is strongly dependent on the model design and on the pressure ratio. El Sayed et al. (2018) quantified this bending for the stainless-steel water tunnel model for a range of pressure ratios using an optical as well as a mechanical approach. Recently (Wierach et al. 2020) measured the lip deflection using an accurate Digital Image Correlation (DIC) on the significantly more plastic ${ }^{2}$ wind tunnel model. Based on these results, we estimate the lip height uncertainty from bending for a typical design to be $b_{S, 2}^{h} \approx 5 \%$. Using equation (17), the two setup uncertainties of the slot height are combined to yield $b_{S}^{h} \approx 11 \%$.

It is possible to reduce the lip height uncertainty using alternative actuator design. Examples include multiple plena (Oswald et al. 2019; Scholz et al. 2020) instead of a single one, and more adjustment bolts through the lid, rendering the lid less susceptible to bending. Such designs can reduce the slot height uncertainty to an estimated $b_{S}^{h} \approx 5 \%$. These uncertainties are denoted in red in Table 2. Personal experience suggests lip height uncertainty lower than 5\% for Coanda actuators with a high aspect ratio jet slot is not achievable. We also caution against some designs that might exchange lower lip height uncertainty for significantly smaller plenum volume, where the isentropic expansion assumption is violated.

The second setup uncertainty is that of the jet exit pressure, which consists of two main error sources: the pressure sensor position, and spanwise pressure distribution. Uncertainty in positioning the pressure taps at the jet exit creates large uncertainty in the sensor readings, which is caused by the steep pressure gradient in the contraction section, as shown in Fig. 5 for the current configuration ${ }^{3}$. Here, $d s^{*}$ is the normalized curvilinear deviations from the true jet exit location $s_{\mathrm{j}}$, defined as,

$d s^{*}=\frac{s-s_{\mathrm{j}}}{c}$.

The red line marks the uncertain pressure range based on the pressure tap estimated position uncertainty of $\pm 0.2 \mathrm{~mm}$ at the jet exit. This position uncertainty is estimated from errors in the pressure tap drill position through the model surface, and errors in the actuator lid position relative to the pressure tap.

The presented pressure distribution in Fig. 5 is generated from numerical simulation of the flow (Semaan et al. 2016). The distribution shows the static pressure around the jet exit spanning the last part of the actuator nozzle and the first bit of the Coanda surface. As the figure shows, slight

\footnotetext{
2 The increased plasticity is due to the individually-controlled 33 deformable lip segments that enable spanwise wave actuation.

3 This issue is irrelevant for actuators without a Coanda surface, where the jet exit pressure can be safely assumed to be that of the free stream.
}

Table 3 Propagated expanded relative uncertainty of the six momentum coefficient definitions

\begin{tabular}{llll}
\hline & $U_{C_{\mu}}^{(1)}[\%]$ & $U_{C_{\mu}}^{(2)}[\%]$ & $U_{C_{\mu}}^{(3)}[\%]$ \\
\hline$C_{\mu 1}$ & 34.96 & 26.10 & 13.18 \\
$C_{\mu 2}$ & 45.69 & 37.20 & 18.70 \\
$C_{\mu 3}$ & 37.66 & 31.91 & 16.38 \\
$C_{\mu 4}$ & 30.07 & 22.45 & 11.36 \\
$C_{\mu 5}$ & 64.03 & 50.00 & 25.01 \\
$C_{\mu 6}$ & 22.72 & 22.72 & 11.88 \\
\hline
\end{tabular}

The superscripts (1), (2) and (3) denote the scenarios with one exit pressure sensor, four exit pressure sensors, and combined four exit pressure sensors with a rigid actuator design, respectively. The results are generated for a fixed pressure ratio of PR $=1.5$

misplacement of the pressure tap leads to significant error in the pressure readings. This is particularly problematic when the sensor location is inside the nozzle, where the pressure gradient is large. Bootstraping (Efron and Tibshirani 1985) pressure samples from the uncertain range (the red line) 10,000 times for the mean yields the probability density function in Fig. 6 and subsequently the systematic setup uncertainty of the jet exit pressure that stands at $b_{S, 1}^{P_{\mathrm{j}}} \approx 10 \%$.

One possible solution to minimize $b_{S, 1}^{P_{\mathrm{j}}}$ is to rely on multiple sensors at the jet exit instead of on only one.

In this study, we consider the scenario of four equallyspaced pressure sensors centered around the real jet exit. As illustrated in Fig. 7, we compute $P_{\mathrm{j}}$ from the intersection of the two linear fits (dashed lines) of the two left and two right sensor readings. $b_{S, 1}^{P_{\mathrm{j}}}$ is then estimated from the probability density function of 2000 intersection point samples generated from randomly sampling the four pressure sensors from their corresponding uncertain range. We note that the uncertain position range of each of the four pressure sensors is the same as that of the single sensor. Despite its simplicity, this approach significantly reduces the jet exit pressure uncertainty to $b_{S, 1}^{P_{j}} \approx 0.05 \%$. In Sect. 4, we shall detail the impact this uncertainty reduction has on that of the momentum coefficient.

The second jet exit pressure setup uncertainty stems from spanwise pressure fluctuations caused by the slot height variations. As shown in Fig. 4, the slot height spanwise variation is also mirrored in the jet exit pressure distributions. Moreover, the two uncertainties have similar magnitude, such that $b_{S, 2}^{P_{j}} \approx b_{S}^{h}$. This suggests that any reduction in the lip height fluctuations is directly reflected in the pressure exit pressure distribution. Combining both setup uncertainties yield $b_{S}^{P_{j}}=11.18 \%$ and $5.59 \%$ for the standard and the improved slot design, respectively. 
Table 4 Uncertainty percentage contributions (UPC) of the measured variables for the six momentum coefficient definitions

\begin{tabular}{|c|c|c|c|c|c|c|}
\hline (1) & $C_{\mu 1}$ & $C_{\mu 2}$ & $C_{\mu 3}$ & $C_{\mu 4}$ & $C_{\mu 5}$ & $C_{\mu 6}$ \\
\hline$\dot{m}$ & 0.33 & 0.00 & 1.14 & 0.45 & 0.00 & 3.13 \\
\hline$T_{\mathrm{j}}$ & 0.00 & 0.11 & 0.16 & 0.00 & 0.00 & 0.00 \\
\hline$T_{\mathrm{pl}}$ & 0.05 & 0.11 & 0.00 & 0.00 & 0.00 & 0.00 \\
\hline$P_{\mathrm{j}}$ & 99.62 & 75.82 & 63.45 & 99.54 & 87.80 & 0.00 \\
\hline$P_{\mathrm{pl}}$ & 0.00 & 0.01 & 0.00 & 0.01 & 0.01 & 0.00 \\
\hline$P_{\infty}$ & 0.00 & 0.00 & 0.00 & 0.00 & 0.00 & 0.00 \\
\hline$h$ & 0.00 & 23.95 & 35.25 & 0.00 & 12.19 & 96.87 \\
\hline$b_{h, P_{\mathrm{i}}}$ & 0.00 & 0.00 & 0.00 & 0.00 & 0.00 & 0.00 \\
\hline (2) & $C_{\mu 1}$ & $C_{\mu 2}$ & $C_{\mu 3}$ & $C_{\mu 4}$ & $C_{\mu 5}$ & $C_{\mu 6}$ \\
\hline$\dot{m}$ & 0.59 & 0.00 & 1.59 & 0.80 & 0.00 & 3.13 \\
\hline$T_{\mathrm{j}}$ & 0.00 & 0.16 & 0.22 & 0.00 & 0.00 & 0.00 \\
\hline$T_{\mathrm{pl}}$ & 0.08 & 0.16 & 0.00 & 0.00 & 0.00 & 0.00 \\
\hline$P_{\mathrm{j}}$ & 99.32 & 63.54 & 49.10 & 99.18 & 79.99 & 0.00 \\
\hline$P_{\mathrm{pl}}$ & 0.01 & 0.02 & 0.00 & 0.02 & 0.02 & 0.06 \\
\hline$P_{\infty}$ & 0.00 & 0.00 & 0.00 & 0.00 & 0.00 & 0.00 \\
\hline$h$ & 0.00 & 36.12 & 49.09 & 0.00 & 20.00 & 96.87 \\
\hline$b_{h, P_{\mathrm{j}}}$ & 0.00 & 0.00 & 0.00 & 0.00 & 0.00 & 0.00 \\
\hline (3) & $C_{\mu 1}$ & $C_{\mu 2}$ & $C_{\mu 3}$ & $C_{\mu 4}$ & $C_{\mu 5}$ & $C_{\mu 6}$ \\
\hline$\dot{m}$ & 2.32 & 0.00 & 6.02 & 3.13 & 0.00 & 11.45 \\
\hline$T_{\mathrm{j}}$ & 0.00 & 0.64 & 0.84 & 0.00 & 0.00 & 0.00 \\
\hline$T_{\mathrm{pl}}$ & 0.32 & 0.64 & 0.00 & 0.00 & 0.00 & 0.00 \\
\hline$P_{\mathrm{j}}$ & 97.32 & 62.89 & 46.57 & 96.80 & 79.95 & 0.00 \\
\hline$P_{\mathrm{pl}}$ & 0.03 & 0.07 & 0.00 & 0.07 & 0.06 & 0.00 \\
\hline$P_{\infty}$ & 0.00 & 0.00 & 0.00 & 0.00 & 0.00 & 0.00 \\
\hline$h$ & 0.00 & 37.75 & 46.57 & 0.00 & 19.98 & 88.55 \\
\hline$b_{h, P_{\mathrm{i}}}$ & 0.00 & 0.00 & 0.00 & 0.00 & 0.00 & 0.00 \\
\hline
\end{tabular}

The numbers (1), (2) and (3) denote the scenarios with one exit pressure sensor, four exit pressure sensors, and combined four exit pressure sensors with a rigid actuator design, respectively. The results are generated for a fixed pressure ratio of $\mathrm{PR}=1.5$

So far, we have neglected any possible correlation among the uncertainties. However, as we have just seen, slot height spanwise variations are strongly correlated with the $P_{\mathrm{j}}$ distributions.

Following Coleman and Steele (2009), we estimate this correlated systematic uncertainty as,

$b_{h, P_{\mathrm{j}}}=b_{S, 2}^{P_{\mathrm{j}}} b_{S, 1}^{h}+b_{S, 2}^{P_{\mathrm{j}}} b_{S, 2}^{h}$,

where $b_{S, 1}^{h}, b_{S, 2}^{h}$ and $b_{S, 2}^{P_{\mathrm{j}}}$ are the elemental systematic error sources of $h$ and $P_{\mathrm{j}}$ that are correlated. The values of $b_{h, P_{\mathrm{j}}}$ are obviously dependent on how stiff the actuator lid is. For the considered scenarios, $b_{h, P_{\mathrm{j}}}$ varies from 1.67 to $0.42 \%$ between the the standard and the stiff design, respectively. Considering that only $h$ and $P_{\mathrm{j}}$ have correlated uncertainties, equation (12) becomes,
$u_{C_{\mu}}^{2}=\sum_{i=1}^{I}\left(\frac{\partial C_{\mu}}{\partial x_{i}}\right)^{2} u_{x_{i}}^{2}+2\left(\frac{\partial C_{\mu}}{\partial h}\right)\left(\frac{\partial C_{\mu}}{\partial P_{\mathrm{j}}}\right) u_{h, P_{\mathrm{j}}}$.

As shown in Table 4, where the uncertainty percentage contributions are listed, the contribution of this correlated uncertainty is negligible for all momentum coefficient definitions and for all scenarios. In other words, all measured variables can be assumed statistically independent.

\subsection{Assumptions}

Before we present and discuss the results, we first summarize and list all the assumptions we have so far made. This is to delineate the limitations and the applicability range of the current analysis. For example, some of the drawn conclusions from the current benchmark example do not apply (e.g. lip height uncertainty for mirco jets) or do not extend 
far enough (e.g. unsteady blowing) to other fluidic actuators. For the current analysis, we assume the following:

1. The jet exit velocity is constant across the slot height.

2. The jet exit velocity is subsonic.

3. We exclude the scenario of measuring $V_{\mathrm{j}}$ directly. Direct velocity measurements at the jet exit disturb the flow and diminish the Coanda effect.

4. We only consider steady blowing.

5. The uncertainty of the plenum temperature measurement is similar to that of at the jet exit, $u^{T_{\mathrm{pl}}} \approx u^{T_{\mathrm{j}}}$.

6. The plenum is large enough to yield a quasi-zero velocity in the plenum, $V_{\mathrm{pl}} \approx 0 \mathrm{~m} / \mathrm{s}$.

7. The nozzle efficiency factor $\xi$ has negligible uncertainty.

8. The angular systematic uncertainty of the pitot Prandtl tube measuring $P_{\infty}$ is negligible.

9. For incompressible flow, the flow density uncertainty is negligible, $u^{\rho_{\infty}} \approx 0$.

10. The data reduction equation (11) is not highly nonlinear.

11. We are only interested in propagating the uncertainty of the mean values.

12. All uncertain variables are statistically independent except for setup systematic uncertainties of $h$ and $P_{\mathrm{j}}$.

13. The results are presented for a $95 \%$ confidence level.

14. We acquire large sample records.

15. Proper and accurate usage of acquisition hardware and careful calibration is conducted.

\section{Results}

In this section, we present the uncertainty of the six momentum coefficient definitions after propagating the different random and systematic uncertainties using equation (19). The results are generated for a fixed pressure ratio of PR $=1.5$, which is in the mid-subsonic pressure ratio range. Table 3 lists the propagated expanded relative uncertainty of the six momentum coefficient definitions. The superscripts (1), (2) and (3) denote the scenarios with one exit pressure sensor, four exit pressure sensors, and combined four exit pressure sensors with a stiff actuator design, respectively. The MATLAB code used for the analysis is freely available under https://github.com/rsemaan/UQ_Cmu.

For the first scenario with only one sensor at the exit, the uncertainty of all momentum coefficient definitions is very elevated. The smallest uncertainty is that of $C_{\mu 6}$ at $22.72 \%$, whereas the highest is that of $C_{\mu 5}$ at a whopping $64.03 \%$. The largest uncertainty contributors can be identified in Table 4, where the UPC's for all six definitions and three scenarios are listed. As the table shows, except for $C_{\mu 3}$ and $C_{\mu 6}$ where $P_{\mathrm{j}}$ is either not a dependent variable or plays a minimal role, most of the uncertainty stems from the jet exit pressure measurements. The large uncertainty is a result of a compounded effect of high UMFs and high uncertainty of the jet exit measurements. The uncertainty magnification factors are direct byproducts of the data reduction equation. As such, there is no possibility to reduce them. Significant effort should, therefore, be undertaken to reduce $U^{P_{\mathrm{j}}}$.

Scenario (2) offers the first effort to reduce the uncertainty of $U^{P_{\mathrm{j}}}$ and hence of $C_{\mu}$. The scenario employs four sensors near the jet exit to compute $P_{\mathrm{j}}$. We emphasize that neither the proposed number of sensors nor the method to infer $P_{\mathrm{j}}$ from the linear intersection are strict requirements. Many other methods with a different number of sensors are foreseeable. The important takeaway is that a single uncertain sensor at the jet exit is not sufficient to deliver reliable momentum coefficient estimates for Coanda actuators. As Table 3 shows, the relative expanded uncertainties of $C_{\mu}$ are now reduced for all six definitions (except for $C_{\mu 6}$, which is independent of $P_{\mathrm{j}}$ ). The momentum coefficient uncertainty for the four other definitions remains dominated by $U^{P_{\mathrm{j}}}$. This is confirmed by the uncertainty percentage contributions in Table 4, where the jet exit uncertainty contributes with at least $49.90 \%$ to the total uncertainty. Despite the improvements, the relative uncertainty of $C_{\mu}$ remains high.

Further reduction in the uncertainty can be achieved through a more robust and improved design of the actuator slot. Such designs can reduce the relative setup systematic uncertainty of the lip height and of the jet exit pressure to $b_{S}^{h} \approx b_{S, 2}^{P_{\mathrm{j}}} \approx 5 \%$. This reduction, in turn, lowers the uncertainty of $C_{\mu}$. Combined measurement approaches relying on both mass flow and pressure measurements become much more favorable with $U_{C_{\mu}}^{(3)}=13.18$ and $11.36 \%$ for $C_{\mu 1}$ and $C_{\mu 4}$, respectively. We recall the negligible uncertainty assumption on the nozzle efficiency factor $\xi$. Failure to accurately estimate $\xi$ might lead to large uncertainty that will eventually propagate into $C_{\mu}$. Massflow measurement approaches are similarly attractive with $U_{C_{\mu}}^{(3)}=16.38$ and $11.88 \%$ for $C_{\mu 3}$ and $C_{\mu 6}$, respectively.

Despite the two suggested improvements, the uncertainty of the momentum coefficient for a Coanda actuator with a high aspect ratio slot remains considerable. As listed in Table 4, the main uncertainty contributor is $P_{\mathrm{j}}$ followed by $h$. In the author's opinion, further significant uncertainty reductions for this actuator type are not feasible. Any reporting of active flow results employing such actuators should therefore include the uncertainty margins. 


\section{Conclusions and recommendations}

In this study, we perform uncertainty quantification analysis on six different momentum coefficient definitions for various blowing conditions and experimental settings. The uncertainty is propagated from the various measured variables to $C_{\mu}$ using a first-order Taylor expansion method. The analysis employs numerical and experimental data on a high-lift configuration equipped with a Coanda flap. All relevant random and systematic uncertainties of the measured variables are quantified and listed. For a single pressure sensor at the exit with standard actuator design, the results show unacceptably high uncertainty for all six momentum coefficient approaches. The elevated uncertainties are traced to a combination of high uncertainty magnification factors for the jet exit and the plenum pressures, and to two high measurement uncertainties: the jet exit pressure and the lip height. Two possible solutions to reduce these two large uncertainty contributors are presented and analyzed. The two solutions consist of an improved jet exit pressure estimation method and more rigid actuator design. These improvements are shown to significantly reduce the uncertainty of the momentum coefficient. The combined and mass flow measurement approaches yield the lowest uncertainties of $C_{\mu}$. However, even with the proposed improvements, the most accurate approach still yields a relative expanded uncertainty of $\approx 11 \%$. It is, therefore, crucial to include the uncertainty margins when reporting results that employ this and similar actuators. Here, we acknowledge the associated experimental challenges in measuring particular quantities (e.g., $T_{\mathrm{j}}$ ), and the limited accuracy of some commercially-available equipment (e.g., mass flow meter), which constitute a lower bound to the achievable uncertainty.

We also note that lower uncertainty of $C_{\mu}$ can be achieved for different fluidic actuators designs, such as micro-jets and distributed slots, where the jet exit pressure and the slot height distribution uncertainties are much smaller or even negligible.

Another objective of this study is to provide recommendations on how to best measure the momentum coefficient. Based on the achieved results, and taking into account the listed assumptions (c.f. Sect. 3.4), the following general recommendations (applicable to all fluidic actuators) are inferred:

1. Identify with certainty whether the jet exit flow is compressible or incompressible. In case of doubt, assume compressible flow.

2. For steady blowing, the so-called combined approach employing mass flow rate and pressure measurements is the most accurate. This suggestion is valid when the nozzle efficiency factor $\xi$ is accurately estimated, which can be achieved through numerical simulation, from existing catalog listings, or can be estimated (with possibly larger uncertainty) by simply equating $C_{\mu 1}$ to $C_{\mu 3}$ (or $C_{\mu 4}$ to $C_{\mu 6}$ for incompressible flows). Alternatively, $\xi$ can be estimated at the beginning of a measurement campaign by careful measurements of the jet exit velocity distribution.

3. For unsteady blowing with relatively high actuation frequency, the pressure-based methods $\left(C_{\mu 2}\right.$ and $\left.C_{\mu 5}\right)$ are recommended, since they can deliver a real-time response. This limitation stems from the slow response time of mass flow meters.

4. Extra care should be taken when estimating the momentum coefficient at low-pressure ratios, where the uncertainty magnification factors are large.

5. All experimental data should be acquired sufficiently long to ensure statistical convergence, and to make use of the large-sample assumption.

6. All measured variables can be assumed statistically uncorrelated.

7. We recommend to use the so-called direct method (Coleman and Steele 2009) to compute the standard deviation of the mean momentum coefficient $\sigma_{\bar{C}_{\mu}}$ for the random uncertainty. This direct method bypasses the need to propagate the random uncertainties. It is advantageous when the variables $x_{i}$ have a common, time-varying error source. The random error correlation effects will then automatically be taken into account.

8. Quantify and report the uncertainty margins of the estimated momentum coefficient regardless of how high they may be.

For Coanda actuation with high slot aspect ratio, two additional recommendations are provided:

1. It is recommended to use multiple sensors near the jet exit location to minimize the measurement uncertainty. If multiple sensor deployment at the jet exit is not possible, a reasonable workaround is to assume an isotropic expansion to free stream pressure $P_{\infty}$, which has been shown to yield $\mathrm{a} \approx 6.3 \%$ error at $\mathrm{PR}=2$ for the wind tunnel experiment (El Sayed et al. 2018).

2. The lip height distribution is a significant uncertainty contributor to all $C_{\mu}$ definitions. It is, therefore, crucial to:

(a) accurately measure the spanwise slot height distribution using a laser-based scanner.

(b) make sure the spanwise slot height and consequently the jet exit pressure distributions do not deviate more than $10 \%$ from the nominal value. This step does not only reduce the related uncer- 


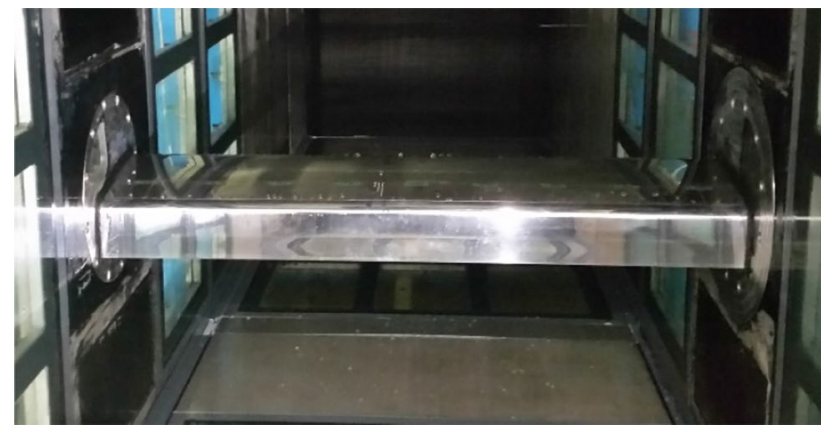

Fig. 8 The stainless steel experimental model installed in the water tunnel

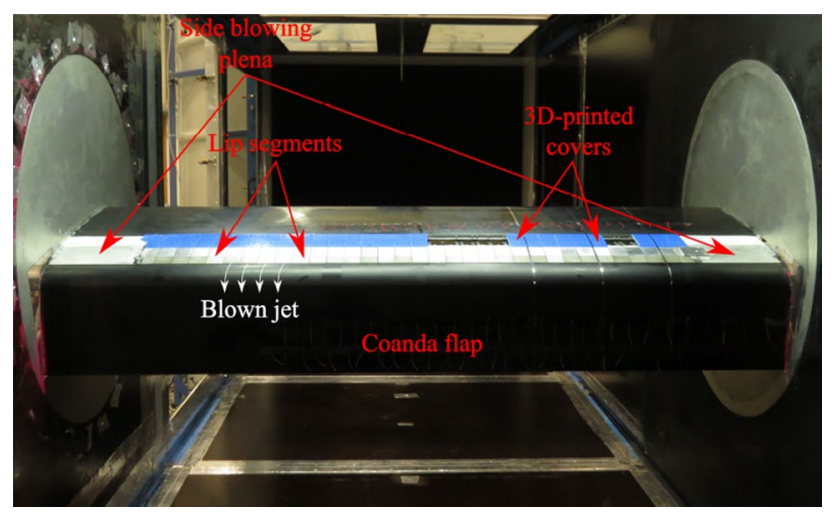

Fig. 9 The carbon fiber composite experimental model installed in the wind tunnel. Denoted are the blown jet location, the individuallycontrolled lip segments, the actuator covers, and the side-blowing plena

tainties but also ensures the flow is homogeneous in the spanwise direction.

(c) design a rigid lid that is resistant to bending during operations.

\begin{abstract}
Acknowledgements We acknowledge the funding and excellent working conditions of the Collaborative Research Centre (CRC880) 'Fundamentals of High Lift of Future Civil Aircraft' supported by the Deutsche Forschungsgemeinschaft (DFG) and hosted at the Technische Universität Braunschweig.
\end{abstract}

Funding Open Access funding enabled and organized by Projekt DEAL. This study is supported by Deutsche Forschungsgemeinschaft (Grant No. SFB 880).

Open Access This article is licensed under a Creative Commons Attribution 4.0 International License, which permits use, sharing, adaptation, distribution and reproduction in any medium or format, as long as you give appropriate credit to the original author(s) and the source, provide a link to the Creative Commons licence, and indicate if changes were made. The images or other third party material in this article are included in the article's Creative Commons licence, unless indicated otherwise in a credit line to the material. If material is not included in the article's Creative Commons licence and your intended use is not permitted by statutory regulation or exceeds the permitted use, you will need to obtain permission directly from the copyright holder. To view a copy of this licence, visit http://creativecommons.org/licenses/by/4.0/.

\section{Appendix 1: Numerical setup}

Part of the current investigations is based on a two-dimensional numerical simulation of an airfoil with a Coanda flap. In this appendix, we present the details of the benchmark high-lift configuration and the numerical setup.

\section{Configuration}

The high-lift configuration, shown in Fig. 1 is a modified DLR F15 airfoil equipped with a highly deflected Coanda flap and a droop nose. The details of the airfoil design are described in Burnazzi and Radespiel (2014) and Jensch et al. (2009), where the objective was to accomplish high lift coefficients during take-off and landing. The leading edge geometry was reached after an iterative process that improved the airfoil stall behavior, which is ruled by the suction peak. The highly deflected flap at $65^{\circ}$ has a chord length of $c_{\mathrm{ff}}=0.25 \mathrm{c}$. The flap is specially designed with a round shoulder to enhance the Coanda effect. The numerical simulations are performed at Mach number $\mathrm{Ma}=0.15$, and Reynolds number $\operatorname{Re}=U_{\infty} c / v=12 \cdot 10^{6}$, where $v$ is the kinematic viscosity of the fluid. These flow parameters correspond to the expected conditions during landing.

\section{Unsteady Reynolds-Averaged Navier-Stokes simulations}

The CFD solver employed to perform the analysis is the DLR TAU code (Schwamborn et al. 2006). The two-dimensional Unsteady Reynolds Averaged Navier-Stokes (URANS) equations are solved using a finite volume approach. The discretization schemes are the central differencing scheme and the second order upwind Roe scheme for the mean-flow inviscid flux and the convective flux of the turbulence transport equation, respectively. The turbulence model is that of Spalart-Allmaras with curvature correction, which allows the one-equation turbulence model to maintain a good accuracy in regions where the streamlines have a high curvature. This characteristic is fundamental for the simulation of the Coanda phenomenon, which is based on the equilibrium between the inertial forces and the momentum transport in the direction normal to the convex surface.

The mesh is composed of a structured and an unstructured region comprising about 230,000 grid points. The structured 
grid extends from the airfoil surface outward to cover the region where the main viscous phenomena occur. The viscous sub-layer is also resolved with $y_{+}<1$ everywhere over the airfoil surface. An important characteristic of the grid is the high grid density along the pressure side, where the stagnation point can be located, far from the leading edge. The structured region is also extended over a large area behind the highly-deflected flap, to accurately capture the wake dynamics during the separated non-actuated period. Both, the trailing edge and the edge of the slot lip, are discretized using a local C-block topology.

\section{Appendix 2: Experimental setups}

The utilized experimental data are provided from two experimental measurements in the wind and the water tunnel. This section describes both models, the facilities, and the instrumentation and measurement techniques.

\section{Models}

The water tunnel experimental model shown in Fig. 8 has a chord length of $c=300 \mathrm{~mm}$ and a span of $1 \mathrm{~m}$. The Coanda-flap has a length of $c_{\mathrm{fl}}=0.25 \cdot c$ (i.e. $c_{\mathrm{fl}}=75$ $\mathrm{mm})$ and is deflected by $65^{\circ}$ for a landing configuration. The Coanda jet is blown over the flap shoulder through a $0.00067 \cdot c=0.2 \mathrm{~mm}$ slot. The jet is supplied through a plenum inside the model, which is connected on both sides of the model to a KSB Movitec VF 32-7 PD multi-stage inlinepump installed outside the tunnel that delivers flow rates up to $10 \mathrm{l} / \mathrm{s}$ at 8 bar pressure.

The wind tunnel experimental model is the same twodimensional modified DLR-F15 airfoil (Fig. 9) but adapted and scaled for the wind tunnel. It has a chord length of $c=600 \mathrm{~mm}$, a $1.3 \mathrm{~m}$ span, and a flap length of $c_{\mathrm{fl}}=150$ $\mathrm{mm}$. The jet is supplied through a plenum inside the model, which is connected on both sides of the model to a PoleStar PST120 refrigeration air dryer installed outside the tunnel and the building compressor, which can deliver flow rates up to $1000 \mathrm{l} / \mathrm{s}$ at $3 \mathrm{bar}$ pressure. To minimize side wall effects, the flow is kept attached near the tunnel walls using two separate Coanda actuation from a separate pneumatic feed.

\section{Facilities}

The water tunnel experiment is carried out in the Großer Wasserkanal Braunschweig (GWB) at the Technische Universität Braunschweig. The facility is a Göttingen-type closed return tunnel, with a $6 \mathrm{~m}$ long and $1 \mathrm{~m} \times 1 \mathrm{~m}$ test section. The flow is driven by a $1.5 \mathrm{~m}$ diameter one-stage axial pump powered by a variable frequency drive $160 \mathrm{~kW}$ electric motor, yielding flow velocities up to $6 \mathrm{~m} / \mathrm{s}$ in the test section.
To inhibit cavitation, the tunnel can be pressurized up to 2 bar above ambient pressure. Although Reynolds numbers of up to $\operatorname{Re}=\frac{V_{\infty} c}{v_{\infty}}=2.5 \times 10^{6}$ are possible, the Reynolds number in the current study is limited to $1.5 \times 10^{6}$ at $4.5 \mathrm{~m} / \mathrm{s}$ due to concerns about exceeding the load limit of the model.

The wind tunnel experiment is carried out in the "ModellUnterschallkanal Braunschweig" (MUB) at the Institute of Fluid Mechanics of the Technische Universität Braunschweig, which is a low speed, closed-circuit tunnel with interchangeable test sections. The test section used in this study has a cross-section of $1.3 \times 1.3 \times 5$ meters, which allows for a maximum flow velocity of $60 \mathrm{~m} / \mathrm{s}$ when empty. The current experiment is conducted at a reduced velocity of $\approx 50$ $\mathrm{m} / \mathrm{s}$ corresponding to a chord-based Reynolds number of $1.75 \times 10^{6}$. The wind tunnel is driven by a $300 \mathrm{~kW}$ variable speed DC motor. The tunnel temperature is kept constant by a water-cooled heat exchanger in the settling chamber.

\section{Instrumentation and measurement technique}

In the water tunnel experiment, the pressure distributions are measured using 64 pressure taps along the mid-span section connected to high precision ( $0.1 \%$ full-scale error) Keller PD-X33 pressure transducers. Each ensemble is sampled at $100 \mathrm{~Hz}$ for 5 seconds. Besides the pressure taps, the model is instrumented with two real-time miniature piezo-resistive pressure Keller sensors with $300 \mathrm{kHz}$ possible eigenfrequency that are flush mounted onto the Coanda flap. Two Proline Promag 10D Electromagnetic flowmeters are used to measure the mass flow rate.

In the wind tunnel experiment, the pressure signals are collected from 196 surface pressure taps drilled mainly along three spanwise locations. The pressure measurements are acquired using a temperature compensated DTC Initium pressure measurement system with an ESP-64HD pressure scanner. The scanner has a $7 \mathrm{kPa}$ pressure range and a full-scale accuracy of $0.03 \%$. For each measurement 400 samples are acquired at a sampling frequency of 100 Hz. The wake dynamics are captured by eight time-resolved XCQ-093 Kulite pressure sensors distributed around the flap mid-span. The Kulites have a pressure range of $35 \mathrm{kPa}$ and an accuracy of $0.1 \%$ of the full scale. For each measurement 4000 samples are acquired at a sampling frequency of $1 \mathrm{kHz}$. The flow rate is measured using a Testo 6444 flowmeter.

\section{References}

Burnazzi M, Radespiel R (2014) Design and analysis of a droop nose for coanda flap applications. AIAA J Aircr 51(5):1567-1579

Cattafesta LN III, Sheplak M (2011) Actuators for active flow control. Annu Rev Fluid Mech 43(1):247-272 
Coleman HW, Steele GW (2009) Experimentation, validation, and uncertainty analysis for engineers. Wiley, Hoboken

Du X, Chen W (2002) Efficient uncertainty analysis methods for multidisciplinary robust design. AIAA J 40(3):545-552

Efron B, Tibshirani R (1985) The bootstrap method for assessing statistical accuracy. Behaviormetrika 12(17):1-35

El Sayed YM, Semaan R, Sattler S, Radespiel R (2017) Wake characterization methods of a circulation control wing. Exp Fluids 58(10): 144

El Sayed YM, Beck N, Kumar P, Semaan R, Radespiel R (2018a) Challenges in the experimental quantification of the momentum coefficient of circulation controlled wings. New results in numerical and experimental fluid mechanics XI, notes on numerical fluid mechanics and multidisciplinary design. Springer, Berlin, pp 533-543

El Sayed MY, Oswald P, Sattler S, Kumar P, Radespiel R, Behr C, Sinapius M, Petersen J, Wierach P, Quade M, Abel M, Noack BR, Semaan R (2018b) Open- and closed-loop control investigations of unsteady Coanda actuation on a high-lift configuration. AIAA Paper 3684

Englar R (2000) Circulation control pneumatic aerodynamics: blown force and moment augmentation and modification-Past, present and future. AIAA Paper 2000-2541

Englar R, Jones G, Allan B, Lin J (2009) 2-d circulation control airfoil benchmark experiments intended for cfd code validation. In: AIAA Paper 2009-902

François DG, Radespiel R, Semaan R (2018) Numerical investigations of spanwise-varied unsteady coanda actuation on high-lift configuration. J Aircr 55(4):1720-1730

Glezer A, Amitay M (2002) Synthetic jets. Annu Rev Fluid Mech 34(1):503-529

Jensch C, Pfingsten KC, Radespiel R, Schuermann M, Haupt M, Bauss S (2009) Design aspects of a gapless high-lift system with active blowing. In: 58. Deutscher Luft- und Raumfahrtkongress, Aachen, Germany

Kelly MW (1956) Analysis of some parameters used in correlating blowing-type boundary-layer control data. NASA report, RM A56F12

$\mathrm{Ku} \mathrm{HH}$ (1966) Notes on the use of propagation of error formulas. J Res Natl Bur Stand-C Eng Instrum 70C(4): 1

Kumar V, Alvi FS (2006) Use of high-speed microjets for active separation control in diffusers. AIAA J 44(2):273-281

Oswald P, Semaan R, Noack BR (2019) Open- and closed loop control on a D-shaped bluff body equipped with Coanda actuation. AIAA Paper 2019-3601
Padulo M, Campobasso MS, Guenov MD (2007) Comparative analysis of uncertainty propagation methods for robust engineering design. In: International conference on engineering design, ICED'07, Cité des sciences et de l'industrie, Paris France

Pastoor M, Henning L, Noack BR, King R, Tadmor G (2008) Feedback shear layer control for bluff body drag reduction. J Fluid Mech 608:161-196

Poisson-Quinton P, Lepage L (1961) Survey of French research on the control of boundary layer and circulation. Lachmann, GV, "boundary layer and flow control. Princ Appl 1:21-73

Radespiel R, Burnazzi M, Casper M, Scholz P (2016) Active flow control for high lift with steady blowing. Aeronaut J 120(1223):171-200

Raman G, Hailye M, Rice EJ (1993) Flip-flop jet nozzle extended to supersonic flows. AIAA J 31(6):1028-1035

Scholz P, Casper M, Ortmanns J, Kähler CJ, Radespiel R (2008) Leading-edge separation control by means of pulsed vortex generator jets. AIAA J 46(4):837-846

Scholz P, Singh VM, Gebhardt A, Löffler S, Weiss J (2020) The efficiency of different flow control methods on a vertical tail. AIAA paper 2020-1537

Schwamborn D, Gerhold T, Heinrich R (2006) The DLR TAU-code: recent applications in research and industry. In: European conference on computational fluid dynamics, ECCOMAS CFD

Semaan R, Yadav V (2020) SCOUT: signal correction and uncertainty quantification toolbox in MATLAB. SoftwareX, 11

Semaan R, Kumar P, Burnazzi M, Tissot G, Cordier L, Noack BR (2016) Reduced-order modelling of the flow around a high-lift configuration with unsteady Coanda blowing. J Fluid Mech $800: 72-110$

Shaqarin T, Oswald T, Semaan R, Noack B (2019) Closed-loop drag reduction over a D-shaped body via Coanda actuation. In: 5 th symposium on fluid structure-sound interactions and control (FSSIC), Crete island, Greece

Viets H (1975) Flip-flop jet nozzle. AIAA J 13(10):1375-1379

Wierach P, Petersen J, Sinapius M (2020) Design and experimental characterization of an actuation system for flow control of an internally blown coanda Flap. Aerospace 7(3):1

Publisher's Note Springer Nature remains neutral with regard to jurisdictional claims in published maps and institutional affiliations. 